\title{
Sampling techniques procedures in sustainable forest management; a two-phase sampling approach
}

\section{Oyamakin Oluwafemi $\mathbf{S}^{*}$., Julius Adebayo J., Asaolu Janet O. and Oyeleye Bola}

\author{
Forestry Research Institute of Nigeria, P.M.B. 5054, Jericho, Ibadan. \\ "e-mail: fm_oyamakin@yahoo.com, +2348066266535 \\ ABSTRACT
}

\begin{abstract}
In other to address the problem of cost, time and precision of research activities in sustainable forest management, a simple random sub-sample $S$ of size $\mathrm{n}$ is then taken without replacement from $S^{\prime}$ and the characteristic of interest $y_{i}$ is observed for all $\mathrm{i} \in S$. The data used for this work are the records of the measure of the girth and height of teak (Tectona grandis) trees based on a survey (thinning exercise) carried out at the Forestry Research Institute of Nigeria, Ibadan in 2007. The records indicated an estimated total of about 1000 (one thousand) teak trees on this plantation. A first phase sample S' of size 80 was taken with the measures of the girth (in metres) of the tree as auxiliary variable and also a second phase sample (subsample) S of size 40 was taken from the first phase sample S' giving the measure of the height (in metres) of the study variable $y$. This study shows that the method of two-phase sampling is a powerful and economical technique in survey sampling as it yields a gain in precision in the estimation of population characteristics given specified cost limits as against that of simple random sampling.
\end{abstract}

Keywords: simple random sampling, thinning, tectona grandis, two-phase sampling, girth, height

\section{INTRODUCTION}

In survey sampling inquiries, the investigator is usually faced with constraints in terms of; cost, precision, practicability, time etc. This must be taken into consideration in the design of the sampling plan. In practice, it is often the case that the characteristic that is of principal interest to the investigator may be very expensive to measure. However another characteristic can be identified which is highly correlated with the first one and relatively inexpensive to measure. These characteristics will be referred to as the study variable $y$ and the auxiliary variable $x$, respectively. Many sampling schemes depend on the possession of information about the auxiliary variable $x$, or a vector of auxiliary variables available for the entire population.

For estimating the mean of $y$, a single sampling plan which takes observations only on $y$ may not yield an estimator with desired precision, since it may not be feasible to take a sufficiently large number of observations because of budget constraints. Therefore where information on the auxiliary variable is not available we adopt the technique of two-phase sampling without appreciably adding to the cost of the survey. Neyman considered this problem in detail, developed and presented the theory of double sampling (also known as two-phase sampling) Neyman (1938). In that paper Neyman also introduced explicit cost function thereby introducing the joint use of both cost and variance function to optimize sample design. Therefore where information on the auxiliary variable is not available we adopt the technique of two-phase sampling without appreciably adding to the cost of the survey. Thinning is a tree removal practice that reduces tree density and competition between trees. Thinning concentrates growth on fewer high quality trees, provides periodic income and generally enhances tree vigour. It is based on information gathered in this survey that the forest managers determine which trees are mature enough to be cut down and the ones for which the growth rates are below expectation and need to be removed( i.e. undesired species). Teak trees with botanical name tecona grandis is a genus of tropical hardwood trees in the family lamiaceae native to south and south-east of Asia.

The timber from teak is used in shipbuilding, boat decks and in the manufacture of outdoor furniture and other articles where weather resistance is desired e.g. PHCN poles. Teak (tecona grandis) is used extensively to make doors and window frames as it is resistant to termite attack. 


\section{MATERIALS AND METHODS}

The data used for this work are the records of the measure of the girth and height of teak trees (tecona grandis) based on a survey (thinning exercise) carried out at the Forestry Research Institute of Nigeria, Ibadan in 2007. The records indicated an estimated total of about 1000 (one thousand) teak trees on this plantation. A first phase sample $S^{\prime}$ of size 80 was taken with the measures of the girth (in metres) of the tree as auxiliary variable and also a second phase sample (subsample) $\mathrm{S}$ of size 40 was taken from the first phase sample $S^{\prime}$ giving the measure of the height (in metres) of the study variable $y$.

\section{RESULTS AND DISCUSSION}

- The correlation between the variables of the girth and height of the trees is strong and positive with a value of $\rho=0.874$.

- The estimates of the population mean using the linear regression estimator gives a value of 14.8616.

- The variance of the estimates based on twophase sampling for regression is relatively lower with respect to that of the single sampling plan with or without cost limits. For the optimum variance estimates the value for the two-phase design is 0.08751 as against that of simple random sampling with a value of 0.1178 , given the same assumed cost.

- The relative efficiency of the two-phase sampling plan increases with proportional increase in the correlation values as shown in table 3 . Demonstrating that the stronger the correlation between the $x$ and $y$ variables, the greater the efficiency of the sampling plan with less cost.

Based on the first phase sample the following parameters were estimated as shown in table 1. A second phase sample (subsample) $\mathrm{S}$ of size 40 was taken from the first phase sample $S^{\prime}$ giving the measure of the height (in metres) of the study variable $\mathrm{y}$. Based on the second phase data the following parameters were measured and shown in table 2

Table 1. Descriptive statistics of the first samples

\begin{tabular}{|l|l|l|l|}
\hline $\mathrm{N}$ & Mean & Variance & $\begin{array}{l}\text { Standard } \\
\text { deviation }\end{array}$ \\
\hline 80 & 0.5290125 & 0.0282438 & 0.1680589 \\
\hline
\end{tabular}

Estimation of variance: Given the linear regression estimator for two-phase sampling as;

$\bar{y}_{l r}=\bar{y}+b\left(\bar{x}^{\prime}-x\right)$

Where $\bar{y}=15.0675 \mathrm{~m}$ and $\bar{x}=0.539175$ with $\bar{x}^{\prime}=0.5290125$

Therefore, $\hat{b} \square 20.186$. The coefficient of correlation between the variables is given as; $\hat{\rho}=0.874$ Therefore, $\hat{\rho}^{2}=0.7638$ The coefficient of correlation is calculated using SPSS 10 . The estimate of $\bar{Y}$ is computed using $\hat{\bar{y}}_{l r}=\bar{y}+b\left(\bar{x}^{\prime}-\bar{x}\right)$ such that $\hat{\bar{y}}_{l r}=14.8616 \mathrm{~m}$ The variance estimates for the two-phase sampling plan is;

$V\left(\bar{y}_{t r}\right)=\frac{S_{y}^{2}\left(1-\rho^{2}\right)}{n}+\frac{1}{n^{\prime}} \rho^{2} S_{y}^{2}-\frac{1}{N} S_{y}^{2}$,

$\hat{V}\left(\hat{\bar{y}}_{l r}\right)=0.18922$

Estimation of Optimum Variance: Given an assumed total cost C of the survey as N100.00 and corresponding costs for the two phases of sampling as; $c^{\prime}=N 0.20, c=N 1.00$

To compute for the optimum sample sizes we have;

$$
\begin{aligned}
& n_{0}^{\prime}=\frac{C}{\left(c^{\prime}+c\right)\left[\frac{\left(1-\rho^{2}\right)}{\rho^{2}}\left(\frac{c^{\prime}}{c}\right)\right]^{\frac{1}{2}}} \\
& n_{0}=\frac{C}{\left(c^{\prime}+c\right)\left[\frac{\rho^{2}}{\left(1-\rho^{2}\right)}\left(\frac{c}{c^{\prime}}\right)\right]^{\frac{1}{2}}}
\end{aligned}
$$

And,

Therefore, $n_{0}^{\prime} \square 336$ and $n_{0} \square 21$

Finally the optimum variance is computed using equation:

$V_{0}\left(\bar{y}_{l r}\right)=\frac{S_{y}^{2}\left(\sqrt{c\left(1-\rho^{2}\right)}+\sqrt{c^{\prime} \rho^{2}}\right)^{2}}{C}-\frac{S_{y}^{2}}{N}$

$V_{0}\left(\bar{y}_{l r}\right)=0.08751$

Comparison with single sampling plan: Assuming the same total cost for the survey, the variance estimates for simple random sampling would be given by; $V_{0}(\bar{y})=\frac{c S_{y}^{2}}{C}-\frac{S_{y}^{2}}{N}$. This gives; 
$V_{0}(\bar{y})=\left(\frac{c}{C}-\frac{1}{N}\right) S_{y}^{2}, V_{0}(\bar{y})=\left(\frac{1.00}{100}-\frac{1}{1000}\right) 13.0923$, and $V_{0}(\bar{y})=0.1178$

As such

$V_{0}(\bar{y})>V_{0}\left(\bar{y}_{l r}\right), 0.1178>0.08751$

$0.1178-0.08751>0$

$0.03029>0$

Therefore two-phase sampling plan is more precise because

$\rho^{2}>\frac{4 c c^{\prime}}{\left(c+c^{\prime}\right)^{2}}, \quad 0.7638>\frac{4 \times 1.00 \times 0 . .20}{(1.20)^{2}}$

$0.7638>0.5556$

Relative efficiency: Given a specified cost for twophase sampling and the single sampling plan we compute the asymptotic relative efficiency as;

$R . E=\frac{V_{0}(\bar{y})}{V_{0}\left(\bar{y}_{l r}\right)}=\frac{0.1178}{0.08751} \quad R . E=1.346$

The Relative Efficiency (precision) of the two-phase sampling plan can be further illustrated by choosing some arbitrary values of the correlation coefficient $\rho$ of $\mathrm{y}$ on $x$ to compare it against the ratio of cost per unit of in second sample to cost per unit in first sample i.e. $\frac{c^{\prime}}{C}$.

To compute for relative efficiency of the two-phase sampling for regression we use the formulae

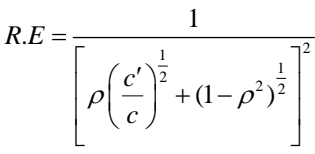

Assigning arbitrary values to $\rho$, which are?

$\rho=0.4,0.5,0.6,0.7,0.8,0.9$

And for the ratio of cost per unit of the first phase sample to cost per unit of second phase sample, $\left(\frac{c^{\prime}}{c}\right)$ which are given as follows;

$c^{\prime}=N 0.00, N 0.01, N 0.05, N 0.10, N 0.50, N 0.80$

And

$C=N 0.50, N 1.00, N 2.00, N 3.00, N 4.00, N 5.00$.

The resulting ratios of $\left(\frac{c^{\prime}}{c}\right)$ are,

$\kappa=0.00,0.01,0.025,0.033,0.125,0.16$. the

resulting values are presented in table 3 .

Table 2. Descriptive statistics of the second samples

\begin{tabular}{|l|l|l|l|l|l|}
\hline $\mathbf{N}$ & Variable & Mean & Variance & Std. Dev. & Sum \\
\hline 40 & $\mathrm{X}$ & 0.539175 & 0.0276744 & 3.681836 & 21.5670 \\
\hline 40 & $\mathrm{Y}$ & 15.0675 & 13.0925 & 0.1663563 & 602.700 \\
\hline
\end{tabular}

Table 3. Relative Efficiency table

\begin{tabular}{|l|l|l|l|l|l|l|l|l|l|l|l|l|}
\hline$\rho$ & $\kappa$ & R.E & $\kappa$ & R.E & $\kappa$ & R.E & $\kappa$ & R.E & $\kappa$ & R.E & $\kappa$ & R.E \\
\hline 0.4 & 0.00 & 1.19 & 0.01 & 1.09 & 0.025 & 1.04 & 0.033 & 1.02 & 0.125 & 0.89 & 0.16 & 0.86 \\
\hline 0.5 & 0.00 & 1.33 & 0.01 & 1.19 & 0.025 & 1.12 & 0.033 & 1.09 & 0.125 & 0.92 & 0.16 & 0.88 \\
\hline 0.6 & 0.00 & 1.56 & 0.01 & 1.35 & 0.025 & 1.25 & 0.033 & 1.21 & 0.125 & 0.98 & 0.16 & 0.93 \\
\hline 0.7 & 0.00 & 1.96 & 0.01 & 1.63 & 0.025 & 1.47 & 0.033 & 1.41 & 0.125 & 1.08 & 0.16 & 1.01 \\
\hline 0.8 & 0.00 & 2.78 & 0.01 & 2.16 & 0.025 & 1.90 & 0.033 & 1.80 & 0.125 & 1.28 & 0.16 & 1.18 \\
\hline 0.9 & 0.00 & 5.26 & 0.01 & 3.62 & 0.025 & 2.99 & 0.033 & 2.78 & 0.125 & 1.76 & 0.16 & 1.58 \\
\hline
\end{tabular}


Am. J. Sci. Ind. Res., 2010, 1(3): 667-670

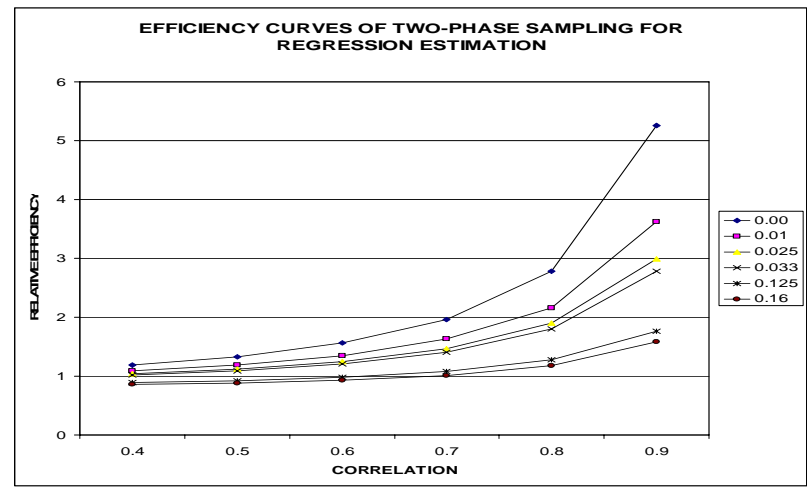

Fig 1: Graph of Relative efficiency against correlation

\section{CONCLUSION}

- The method of two-phase sampling is a powerful and economical technique in survey sampling as it yields a gain in precision in the estimation of population characteristics given specified cost limits as against that of simple random sampling.

- For further study one would suggest the use of two-phase sampling for regression for more than one occasion such that change over time intervals could be estimated.

\section{REFERENCES}

1. Bose, C. 1943 , Note on the Sampling Error in the Method of Double Sampling. Sankhya; Indian Journal of Statistics 6: 329-330.
2. Chen, J. and Rao, J.N.K. 2007,. Asymptotic Normality under Two-phase Sampling designs. Statistica sinica 17:1047-1064

3. Chen, Y.and Chen, H. 2000,. A Unified Approach to Regression Analysis under Double Sampling Designs. Journal of the Royal Statistical Society, series B: $449-460$.

4. Cochran, W.G. 1977,. Sampling Techniques, $3^{\text {rd }}$ Edition New York: Wiley and Sons.

5. Dorfman, A.H. 1994,. A Note on Variance Estimation for the Regression, Estimator in Double Sampling. Journal of the American Statistical Association 89:137-140.

6. El-Beshir, Z.A. 1995,. Non-Response in Double Sampling for Regression. Journal of King Saud $\begin{array}{llll}\text { University } & \text { (Admin.Sci) } & \text { 7: } & 19-36\end{array}$ 